\title{
Dolor crónico y narrativa: experiencias cotidianas y trayectorias de atención en el padecimiento de la migraña*
}

\author{
| ${ }^{1}$ Romina Del Monaco |
}

Resumen: Desde una perspectiva socio antropológica, este trabajo está dirigido a contribuir al conocimiento del padecimiento crónico de la migraña. Para ello se realiza una investigación cualitativa, a través de entrevistas a médicos neurólogos y personas con esta dolencia, en un hospital público del Área Metropolitana de Buenos Aires, Argentina. El objetivo principal es explorar, a través de las narrativas de quienes padecen, las experiencias, prácticas cotidianas y trayectorias de atención con este tipo de dolor de cabeza. Algunas particularidades de la migraña que la diferencian de otras dolencias crónicas, y que condicionan los modos en que los pacientes se relacionan con su entorno residen en que la migraña carece de condiciones fácticas de verificación, y no tiene una etiología precisa ni un tratamiento eficaz. A partir de esto, los sujetos dicen que, en algunos casos, se relaciona al padecimiento con excusas y falta de legitimidad. Por otro lado, a través de los relatos, se observa que la migraña pasa a formar parte de la biografía de la persona. Por último, la mayoría de los pacientes dicen acostumbrarse y resignarse a estos dolores de cabeza y a tener paciencia, algo que influye en la relación consigo mismos y con los otros.

> Palabras-clave: dolor crónico; migraña; experiencias corporales; vida cotidiana; trayectorias de atención.

\author{
1 Becaria doctoral del Consejo \\ Nacional de Investigaciones \\ Científicas y Técnicas \\ (CONICET); Licenciada en \\ Sociología (Universidad de \\ Buenos Aires); Magister en \\ Antropología Social y Política \\ (Facultad Latinoamericana \\ de Ciencias Sociales). Correo \\ electrónico: rominadelmonaco@ \\ yahoo.com.ar
}




\section{Introducción}

En las últimas décadas, las investigaciones sobre dolores crónicos han cobrado una creciente importancia tanto desde la perspectiva biomédica como desde las ciencias sociales. Los fundamentos de estos estudios reposan en que se trata de dolores complejos de ser explicados por la medicina, que se prolongan en el tiempo e intervienen en las vidas cotidianas de las personas que los padecen.

La dificultad biomédica de dar cuenta de estas dolencias se puede explicar (en parte) por tener como modelo epistemológico de referencia a las ciencias biológicas y construir a la enfermedad como un hecho objetivo del cual se desprende un diagnóstico, tratamiento y, en algunos casos, una cura (GOOD, 1994; JACKSON, 2000). Frente a este modelo tradicional, se encuentran las enfermedades crónicas (diabetes, $\mathrm{VIH}$, hepatitis $\mathrm{C}$, etc.) definidas por su prolongación en el tiempo, por ser inciertas en su curso, desarrollo y curación, por ser intrusivas, modificar la vida de los pacientes y por tener un costo elevado (STRAUSS et al., 1984; GRIMBERG, 2003; MARGULIES, 2006). Sin embargo, comparten con las enfermedades más tradicionales el hecho de que, en general, existen evidencias fácticas de su existencia. Por otro lado, existe un conjunto de dolores crónicos tales como la migraña, entre otros, que, si bien la medicina los diagnostica y trata, se diferencian de las enfermedades tradicionales y crónicas, principalmente, porque no hay evidencias "biológicas" de su realidad fáctica y su etiología y explicaciones causales tienden a ser difusas y fragmentarias. Además, son dolencias que trastocan el proceso de síntoma-diagnóstico-tratamiento-alivio y/o cura, instalan nuevos interrogantes a la práctica biomédica y, de acuerdo a la Organización Mundial de la Salud (OMS), se han convertido en causas de numerosas consultas en los servicios médicos en las sociedades actuales.

Los estudios desde la antropología del dolor y sufrimiento acuerdan en que si bien los padecimientos son una condición humana universal, adquieren distintas formas y significados de acuerdo a los contextos y situaciones. Las percepciones y experiencias de padecer son inseparables de influencias sociales, biomédicas, simbólicas, entre otras (GOOD, 1994; KLEINMAN, 1994; JACKSON, 2000). En este sentido, son significativos los aportes de la antropología médica para dar cuenta de las diferencias entre la perspectiva biomédica respecto de procesos de saludenfermedad y aquellas perspectivas abordadas desde las ciencias sociales. El saber biomédico a través de la noción de disease se caracteriza por pensar las dolencias a 
partir de dualismos tales como mente-cuerpo y de considerar a la enfermedad como algo abstracto, una "cosa" que tiene las mismas características independientemente del lugar en el que ocurre dejando de lado las condiciones culturales locales. En cambio, abordar procesos de salud-enfermedad desde la noción de illness reconoce e incluye la perspectiva subjetiva de quienes padecen y, los significados otorgados a dichos acontecimientos, cómo afecta en su comportamiento y en las relaciones con otros (HELMAN, 1984, 1985; KLEINMAN, 1988).

El objetivo de este artículo es indagar en algo en apariencia muy común por hallarse de manera muy frecuente en distintos contextos sociales. Cabe aclarar que si bien existen distintos tipos de dolores de cabeza (migraña, cefalea tensional, cefalea en racimos, cefaleas atribuidas a usos excesivos de medicamente, cefaleas atribuidas a alteraciones craneales, neuralgia trigeminal, etc.) el objetivo de este trabajo es explorar y analizar un dolor de cabeza denominado migraña reconocido y legitimado por la Organización Mundial de la Salud (OMS) como una enfermedad altamente discapacitante.

En este estudio interesa analizar la migraña desde la perspectiva de quienes padecen ya que, si bien el saber biomédico la reconoce como una enfermedad con un diagnóstico y tratamientos (a pesar de las diferencias y contradicciones dentro de la biomedicina respecto de las causas de este dolor de cabeza), la migraña adquiere distintos significados de acuerdo a los contextos sociales en los que se encuentran quienes padecen. Por eso, es importante explorar la cotidianidad y las trayectorias de los sujetos que conviven con un dolor carente de evidencias fácticas vinculado socialmente a algo "común y frecuente".

La gran extensión y presencia de los dolores de cabeza en distintos sectores de la población los convierte en algo poco preocupante y hace que, de acuerdo a las narrativas de los pacientes, parezca no muy importante decir: "tengo migraña". No obstante, en sus experiencias con este dolor, los entrevistados dijeron que éstas incluyen, además de "la cabeza", todo el cuerpo y es un tipo de dolor que los inhabilita para continuar con sus actividades cotidianas. Expresiones en el rostro y acciones como la búsqueda de aislamiento refieren a algo especial que se diferencia de "los dolores de cabeza que tiene todo el mundo". También, distintos estudios epidemiológicos señalan que los dolores crónicos generan grandes gastos en las economías de los países, dado el aumento de la concurrencia periódica de las personas a los servicios de salud. Son dolencias que repercuten en los salarios 
de las personas, no sólo por las faltas recurrentes a sus lugares de trabajo, sino también porque los gastos destinados a tratamientos, costosos en general, ocupan un lugar importante del presupuesto mensual.

Los dolores de cabeza, la migraña, rompen con las explicaciones y los tratamientos tradicionales. Frente al dolor y búsqueda de alivio, las personas tienen distintas respuestas. En algunos casos lo resuelven con recursos cotidianos y/o concurren a los servicios de salud porque dicen que necesitan saber si se trata de algo preocupante y grave. Los saberes expertos "tranquilizan" porque nominan a la dolencia y dan las pautas para una mejoría. No obstante, si bien en la mayoría de las enfermedades (tanto agudas como crónicas), la biomedicina se caracteriza por buscar las causas de los padecimientos en los cuerpos y, a partir de conocimientos objetivos y científicos, realizar un diagnóstico y proponer un tratamiento, este proceso, es diferente en el caso de la migraña.

La migraña pone en evidencia que, para construir el diagnóstico, la biomedicina recupera y reconoce las narrativas subjetivas de los pacientes, sus percepciones y sensaciones de dolor (DEL MONACO, 2012). Además, se realizan estudios tales como tomografías computadas, electroencefalogramas y resonancias para descartar cualquier otra enfermedad. Pero si los resultados se encuentran dentro de los parámetros esperados, es entonces que los profesionales diagnostican "migraña". En otras palabras, la migraña es resultado de que "está todo bien".

El sufrimiento es, fundamentalmente, una experiencia social en distintos aspectos. Implica un vínculo interpersonal complejo con el dolor y con las dificultades de convivir y establecer relaciones sociales con otros a partir de ese padecimiento. Además, se enmarca en términos de aceptación de modelos culturales a los que les corresponden determinadas respuestas morales (KIRMAYER, 2010). La migraña instala un vínculo especial entre las percepciones, sensaciones y emociones de los pacientes, dado que la dolencia se vuelve una constante, algo que se repite e interviene en el mundo de las personas.

En este escrito se define a las nociones de vidas cotidianas y/o modos de vida como las prácticas, acciones, relaciones que tienen las personas y llevan a cabo con cierta frecuencia y regularidad. Se trata de acontecimientos, en general, rutinarios que organizan y permiten cierta previsibilidad.

Por experiencia entiendo un proceso variable, situado en una trama de relaciones inter-subjetivas, construido y reconstruido histórica y socialmente en 
dimensiones normativas, emotivas, valorativas, etc. Las experiencias tensan la relación entre acción y simbolización constituyendo la base de la construcción y el cambio de identificaciones y prácticas sociales (GRIMBERG, 2003). Asimismo, de acuerdo a la perspectiva fenomenológica de Merleau Ponty, retomo la noción de la "experiencia de vivir en el mundo", no como algo pensado sino vivido. Es decir, el mundo se encuentra en la intersección de mis experiencias y las del otro (MERLEAU PONTY, 2003). Estudiar el padecimiento de la migraña como una experiencia de vivir en el mundo permite dar cuenta de procesos complejos que surgen de los vínculos y tensiones entre quien padece y su entorno.

En el análisis de las relaciones del yo con los otros, Butler (2001) analiza cómo en búsqueda de reconocimiento las personas tienden a actuar para los demás y pensando en ellos. Se trata de un yo dinámico, que no sólo es narrado sino también posicionado de diferentes maneras y con diversos propósitos. De acuerdo a esta autora, los otros nos desintegran y, si no fuera así, algo nos faltaría, porque son los lazos que nos ligan con ellos los que nos componen (BUTLER, 2009). El problema surge cuando entre el yo y los otros media un dolor que se prolonga en el tiempo y cuya significación social tiende a ser negativa. Además, dado que socialmente suele vincularse a la cabeza con una parte racional del cuerpo, tener algo en ella puede generar para quienes padecen sensaciones y emociones asociadas a inseguridades, pérdida de control, imposibilidad de comportarse socialmente.

Como se mencionó previamente, este conjunto de sensaciones, experiencias, acontecimientos y vivencias cotidianas en la convivencia con el dolor es lo que Kleinman denomina "illness" para diferenciar la perspectiva de los sujetos que padecen de la noción biomédica de "disease" (KLEINMAN, 1988). La noción de illness permite centrar el interés en los modos en que los relatos de dolor se construyen y adquieren distintos significados y formas teniendo en cuenta que se trata de narrativas particulares en las cuales las experiencias de dolor están mediadas por otros sujetos, por diversos contextos, por preceptos sociales, culturales y morales que intervienen en los modos de experimentar y narrar la convivencia con el padecimiento.

Los relatos de los pacientes crónicos suelen seguir una estructura similar: se inicia con el momento de origen del dolor, luego trayectorias por distintos profesionales, búsqueda de alivio y diagnóstico, realización de varios tratamientos, anécdotas sobre los vínculos entre el padecimiento y las experiencias cotidianas, 
entre otros. El lenguaje entendido como la acumulación objetiva de experiencias y significados (BERGER; LUCKMAN, 1986) continúa siendo el único modo posible de aproximarse a esas sensaciones y expresa - como producto social - la experiencia subjetiva de los individuos, mientras que indexa al orden social que le da origen permitiendo estudiar la relación entre ambos (CASTRO PÉREZ, 2009).

Por eso, examinar las narrativas de los pacientes sobre diversos aspectos de su cotidianidad permite explorar el lugar de mayor o menor importancia destinado a la dolencia. Para eso, se retoma la noción de narrativa como un acto discursivo que presenta una unidad semántica, se refiere a un mundo que pretende describir, expresar o representar (o sea actitudes y comportamientos de los actores), se remite (de forma autorreferencial) a un locutor y se vincula dentro de situaciones de diálogos y performances con un interlocutor (ALVES; RABELO, 2004, 2009).

La biografía de los pacientes con migraña tiende a estar construida a partir de una combinación de eventos dentro de los cuales el dolor ocupa un lugar considerable. Por eso, denomino "biografía del dolor" a las narrativas sobre las características del padecimiento que incluyen, principalmente, relatos cronológicos sobre momentos de la vida atravesados por la dolencia. En este proceso de creación de relatos, la relación y reconocimiento entre el sujeto y los otros es central, porque la constitución del narrador es siempre relacional y la subjetividad se construye a partir de una definición social o "esperada" de sujeto. Abordar el estudio de la experiencia de la migraña desde un enfoque social requiere recuperar los relatos de los pacientes sobre sus formas de sentir y vivir los dolores de cabeza.

En algunos casos, el sufrimiento puede eludir las herramientas lingüísticas y conceptuales y dificultar que las personas puedan entenderse. De acuerdo a Scarry (1985), la resistencia que determinados dolores encuentran al lenguaje también se puede expresar en llantos y gritos. Es decir, en un lenguaje pre-simbólico, que implica cierta destrucción del mundo de esa persona.

En este sentido, estudios sobre el dolor en Antropología aportan una visión del cuerpo como agente de la experiencia (CSORDAS, 1992; GOOD, 1994; DAS, 2001; GRIMBERG, 2003) que permiten explorar cómo se expresan los padecimientos a través de la corporalidad y las emociones en situaciones cotidianas. Asimismo, dan cuenta de las dimensiones sociales de los cuerpos y de la relación recíproca entre lo social y la experiencia corporal, entre los 
aspectos simbólicos del cuerpo de los sujetos y de los contextos sociales, políticos, culturales, etc. (HELMAN, 1988).

A diferencia de otras dolencias/enfermedades crónicas, de la experiencia de la migraña "se entra y se sale". Si bien se trata de un padecimiento caracterizado por crisis recurrentes de dolores de cabeza, mientras no aparezcan esos dolores las personas pueden continuar con sus actividades diarias. La aparición de estos dolores implica retirarse de las tareas habituales y recluirse en espacios aislados para transitar el padecimiento.

Las experiencias, prácticas y acciones corporales sobre sí y por parte de otros involucran la corporización y el "poner el cuerpo", que encuentra en el "aguante" su término de expresión local (EPELE, 2010, p. 232). En investigaciones sobre hinchadas de fútbol, el aguante cumple la función de apoyar, soportar daños (violencia física, molestias, etc.) y se ha convertido en una noción que supone modelos corporales, discursos y códigos de honor (ALABARCES; GARRIGA ZUCAL 2008). En el padecimiento de la migraña el aguante también es social y se orienta a los otros, se trata de un mecanismo central que dicen utilizar las personas para convivir con estos dolores de cabeza a largo plazo.

Analizar la migraña permite explorar un padecimiento que carece de temporalidad específica. Se trata de un dolor que no mata al paciente, cuando se acerca anula el gusto por la vida, pero cuando se aleja se vuelve a la normalidad (ALLUÉ, 2009).

\section{Investigación y métodos}

La investigación adoptó los lineamientos de la metodología cualitativa. El trabajo de campo se desarrolló durante el año 2010 en el servicio de neurología de un hospital público del Área Metropolitana de Buenos Aires (AMBA). Se realizaron 38 entrevistas semi-estructuradas a pacientes con migraña (29 mujeres y 9 varones) de sector socioeconómico medio/medio bajo. El rango etario iba desde los 21 hasta los 60 años.

La guía de preguntas que orientó a las entrevistas semi-estructuradas fueron las siguientes: Condiciones generales de vida: composición familiar, acceso al sistema educativo, trayectorias laborales, accesibilidad y barreras respecto del sistema de salud). Experiencias en relación con el padecimiento: momento en que empezaron los dolores de cabeza, asociación o no con alguna situación particular, 
características de las experiencias corporales y emocionales con el padecimiento, modificaciones del dolor en el tiempo (frecuencia, duración). Modificaciones en las vidas cotidianas a partir de padecimiento: realización de actividades en el tiempo libre, cambio de hábitos y prácticas, reacciones y respuestas de familiares/amigos/pareja ante los dolores de cabeza. Trayectorias y prácticas de cuidado para la migraña: prácticas y estrategias de cuidado, características de los tratamientos, dificultades y obstáculos en sus desarrollos. Se utilizó una guía que se complementó e intercaló con preguntas espontáneas surgidas en el contexto de las entrevistas. La teoría fundamentada guió la construcción de teoría basada en datos empíricos siguiendo un proceso de análisis inductivo.

Resguardos éticos: esta investigación se adecuó a los criterios de consentimiento informado y confidencialidad que se aplican en los estudios sobre salud, con el fin de asegurar los derechos de los/as participantes, así como también de resguardar su identidad. Las personas entrevistadas son mayores de 18 años. Asimismo, para realizar el trabajo de campo en el servicio de salud, se llevó a cabo el proceso de evaluación requerido a través del comité de ética del hospital.

\section{Narrar el dolor}

Todas las personas padecemos dolor en determinados momentos. Sin embargo, esta familiaridad se quiebra al considerar que existen distintos tipos de experiencias con el dolor. Específicamente, la experiencia crónica o cronicidad deja de ser un dolor temporario y se convierte en una condición. Una experiencia recurrente que afecta a las personas y a sus redes vinculares.

En sus relatos, los pacientes manifestaron diferentes formas de actuar frente al padecimiento. Si bien dijeron ser modificados por la dolencia, reconocieron adoptar distintas prácticas frente a ella, a los profesionales y al entorno. Se observan intentos por explicar las sensaciones de dolor y las trayectorias por distintos profesionales. "Desesperación”, "locura”, “agonía”, “oscuridad", "silencio", entre otras, eran algunas de las palabras mencionadas para hacer referencia a los dolores de cabeza.

Tener migraña es un bajón, tener migraña...es un dolor inexplicable. O sea, sentís como que mil agujas se te están clavando en la cabeza; sentís también puntadas, un dolor pulsátil desde un lado o sentís presión sobre la cabeza. No querés que nadie te hable, no querés... te cambia el humor. Es un cambio prácticamente de vida que tomás. (Paula, 26 años). 
Los vínculos entre las narrativas de los pacientes sobre sus trayectorias con el dolor y distintos acontecimientos de la cotidianidad permiten incorporar al estudio de la migraña una noción que denomino "biografía del dolor". Por esta categoría entiendo la inclusión, en la biografía personal de los sujetos, de un conjunto de relatos sobre la dolencia que, teniendo un orden temporal, están relacionados con acontecimientos más o menos significativos de sus vidas cotidianas.

La "biografía del dolor" conforma una suerte de biografía sobre el padecimiento que se hilvana con una biografía general que las personas narran de sí mismas. Estas narraciones sobre distintos momentos de la vida, les atribuyen un orden a los acontecimientos que les permite a los sujetos una ubicación en el mundo. En el acto de narrar, lo dicho sobre las experiencias con la migraña construye un yo particular que surge de las expresiones sobre circunstancias, acciones y eventos fragmentados. La narrativa, que convierte esos fragmentos en un todo coherente y unificado permite, por ejemplo, que los pacientes describan un "momento fundante" de los dolores de cabeza.

Como sostiene Ricoeur, para amalgamar y homogeneizar los relatos se requiere incorporar elementos ficcionales que incrementan la coherencia de esos dichos y los conectan entre sí. Este componente ficcional es posible por la noción de "intriga" definida como una síntesis que organiza elementos heterogéneos (RICOEUR, 1986). La noción de componente ficcional (entendida no como oposición a algo real) le da sentido y coherencia a determinados hechos y situaciones. La construcción narrativa en el presente de una experiencia pasada permite darle a un acontecimiento una realidad espacio-temporal concreta e hilvanarlo con otros hechos de la vida.

Abordar el estudio de la experiencia de la migraña desde un enfoque social requiere recuperar los relatos de los pacientes sobre sus formas de sentir y vivir los dolores de cabeza. A través de los dichos de las personas, es posible determinar tres instancias que estructuran la "biografía del dolor". En la mayoría de los pacientes, hay un "momento fundacional" u "origen del dolor", es decir, se recuerda el año o situación que comenzaron los dolores de cabeza. Luego, los relatos se orientan a las "trayectorias de atención". Con este término se hace referencia a las consultas con distintos profesionales (antes y después del diagnóstico de migraña), a las prácticas de cuidado, manejo de los tratamientos y a los cambios en los modos de vidas. Por último, un aspecto significativo son las teorías de los 
propios pacientes sobre sus dolores y la relación que encuentran entre aquéllos y situaciones cotidianas (recuerdos de la infancia, adolescencia, actualidad), ante las cuáles dijeron sentir angustia, impotencia y acostumbramiento.

\section{Primeras experiencias y trayectorias}

Algunos aspectos de las narraciones sobre las experiencias de los dolores de cabeza guardan similitudes entre sí. Usualmente, las historias comienzan con un momento particular de la vida en el cual surge el dolor. En la mayoría de los casos hay un evento que lo precipita y otros acontecimientos involucrados relacionados con su aparición (GARRO, 1994).

Mirá, yo tengo migraña desde los cuatro años. Yo me acuerdo el día que apareció, me acuerdo porque era el casamiento de uno de mis tíos y entre los nervios por los preparativos y demás, me acuerdo de estar con un dolor de cabeza terrible y que por eso no pudimos ir al casamiento (risa) por eso me acuerdo, nos tuvimos que quedar todos. (Paula, 26 años).

En los dichos de Paula, como en otros casos, es posible reconocer un esfuerzo en delimitar y darle forma al dolor. Esto implica, entre otras cosas, dar cuenta de un origen en tiempo y espacio que forme parte de esa biografía y le dé sentido a la convivencia de la persona con el padecimiento (GOOD, 1994). En otros casos refirieron a diversas experiencias como: "Me acuerdo porque era verano", "era chica, porque yo jugaba con mis primos y cuando me agarraba el dolor me tenía que quedar quieta” o "mi papá está enfermo, es alcohólico así que bueno... ese fue el detonante de mis dolores de cabeza, porque el cuerpo somatiza en algo, y ahí te das cuenta que venís padeciendo nervios y tragando cosas de hace tiempo".

En estos casos, el origen de la migraña no fue un hecho individual y singular, sino que formó parte de una experiencia compartida con otros. Este aspecto demuestra que las narrativas sobre las trayectorias y causas de los padecimientos son, ante todo, una interpretación de una situación específica, de una experiencia socio-personal en un determinado momento que refleja en gran medida la imagen que el individuo tiene de sí mismo. Es decir, una narrativa sobre las causas de una dolencia física o emocional habla mucho más de la subjetividad de la persona que de la dolencia propiamente dicha (CAROSO et al., 2004).

Desde la perspectiva de los propios actores, los malestares no pueden considerarse una "entidad biológica", sino que se transforman en experiencias que 
se constituyen y adquieren sentidos durante las interacciones (ALVES; RABELO, 2009). En esta primera etapa, las personas necesitan ubicar cronológicamente y dar cuenta del momento en que dio inicio su vínculo con el dolor. Esto posibilita incorporar el padecimiento en la biografía personal y, consecuentemente "ordenar" los relatos e hilvanarlos coherentemente con otros.

La segunda etapa consiste en lo que he denominado "trayectorias de atención": las personas recurren a distintos profesionales (traumatólogos, oculistas, clínicos, gastroenterólogos) hasta que llegan a los neurólogos, quienes diagnostican migraña. En estos momentos las personas dan cuenta de un perfil activo en la búsqueda de alivio para los dolores de cabeza. A través de técnicas y procedimientos que son variables, numerosos y se transforman de acuerdo al contexto histórico, los sujetos, en este caso los pacientes con migraña, se producen y surgen de entramados de prácticas y saberes que son atravesados por dispositivos de poder que regulan determinados modos de cuidado de sí (FOUCAULT, 2008; 2010).

Otra cuestión recurrente en los relatos de los pacientes son las percepciones de los demás sobre la migraña. En numerosas oportunidades dicen que se trata de un dolor "mal visto", criticado, cuestionado por el entorno, es decir, estigmatizado.

Es terrible el dolor, a veces la gente no se imagina, te dicen: 'Dolor de cabeza, sí, tomate una aspirina' o 'Ah no querés tener sexo'... un dolor de cabeza, no, no sabés... porque aparte te descompensa todo. (Mirtha, 41 años).

En algunos casos, los sujetos dijeron que sus dolores de cabeza eran considerados (por otros) excusas para no realizar y cumplir con actividades laborales, educativas, familiares, sexuales, entre otras. También se vincula y cuestiona a la migraña como un tipo de queja "común que tiene todo el mundo y se soluciona con una aspirina”. A partir de estas características, algunas de las estrategias que los sujetos dijeron utilizar eran: ocultar el dolor o modificarlo "por uno más creíble".

Por este motivo resulta paradójico que, si bien desde la biomedicina la migraña goza de cierta legitimidad dado que se trata de una enfermedad reconocida (a pesar de las contradicciones observadas dentro de esta práctica respecto de su etiología, tratamientos y modos de prevención), es en distintos contextos sociales donde estos dolores de cabeza encuentran cierta dificultad para ser aceptados como una enfermedad "real". 


\section{Consultas, diagnóstico y más consultas}

Los relatos sobre las formas de actuar ante los dolores de cabeza varían de acuerdo a los actores entrevistados. Los propios pacientes reconocieron convivir durante años con dolores de cabeza hasta decidirse a ver a un profesional. Esto tiene que ver no sólo con las particularidades atribuidas a la migraña, sino también con las dificultades de acceso económicas y sociales a los servicios de salud. Como decía Mercedes, de 25 años, una paciente: “¿Quién no tuvo dolor de cabeza alguna vez?, entonces se piensan que la migraña es eso, no entienden y creen que exagero”.

El detonante de las consultas no es uniforme. En algunos casos, tiene que ver con el aumento de la frecuencia e intensidad de los dolores de cabeza por semana. En otros casos, por las complicaciones laborales y familiares que acarrea o, simplemente por "no aguantar más y necesitar de una respuesta y solución”.

Primero me hicieron placas nasales pensando que era sinusitis, después me sacaron una muela que estaba mal porque pensaban que era por dolor de muelas. Otro, un neurocirujano, por ejemplo, me dijo que era neuralgia y me recetó un medicamento antiepiléptico... o sea, pasé por un montón de médicos y analgésicos y siempre, bueno, me daban una medicación distinta. Y casi siempre me daban antiinflamatorios con relajantes que me estaban destruyendo el estómago. (María, 25 años).

Como en el caso de María, las trayectorias y recorridos incluyen una variedad de profesionales que resume las múltiples formas posibles de abordar los dolores de cabeza (problemas en la columna, mala alimentación, nervios, problemas en la vista, etcétera). También, los sujetos deciden buscar otras prácticas y seguir consejos del entorno con el objetivo de probar distintas alternativas para aliviar el dolor. Dicho aspecto se encuentra exacerbado porque desde el saber biomédico no existen certezas respecto de los motivos que pueden prevenir la aparición de los dolores de cabeza (los posibles "detonantes" del dolor son numerosos e incluyen alimentación, horas de sueño de más o de menos, actividad física por falta o en exceso, estrés, entre otros).

Y..., vi unos cuantos neurólogos antes. Y después he ido a ver a varios médicos clínicos y ahí es donde me hicieron el electroencefalograma, los análisis de sangre. Y después no me acuerdo quién me mandó a hacer la tomografía computada...hasta kinesiólogos he ido a ver porque me dijeron que era por el cuello y demás. Los otros tres neurólogos que fui me dijeron que no había medicación para la migraña, que me cuidara en las comidas y que intente estar tranquila. Qué bueno, así como me viene se me tiene que ir. (Cecilia, 27 años).

... Así que después fui al neurólogo y me dijeron: 'Bueno, tiene que ser neurológico, y sí, es migraña'. Empecé tomando Migral. Pero siempre lo mismo, o sea, me decían 
dormir ocho horas justas'. Pero no se puede eso en la vida real. (Marina, 26 años).

En el caso de Marina, como en la mayoría de los entrevistados, fue luego de concurrir a distintos profesionales que "esos dolores de cabeza que tiene todo el mundo" se transformaron en migraña, una enfermedad definida y delimitada por médicos neurólogos a partir de la reunión de ciertos síntomas y características. No obstante, en otros casos los pacientes dijeron tener migraña desde antes de concurrir a los neurólogos. Esta doble situación da cuenta de la complejidad de un padecimiento cuyo diagnóstico deja de estar sólo a cargo de los profesionales. Es decir, pueden existir dentro del entorno personas con dolores de cabeza similares que comparten información respecto de medicación y prácticas para aliviar el dolor. Incluso, existen personas que se auto definen como "migrañosas" antes de haber consultado a un profesional de la salud.

No obstante, para la mayoría de los entrevistados, la relevancia del momento del diagnóstico (biomédico) se debe a que la incertidumbre y angustia tienden a ser remediadas por la palabra oficial de la medicina. Dar nombre al padecimiento permite que la dolencia se encuentre inscripta en categorías que dan la expectativa de vivir en un mundo ordenado donde la condición del padecimiento es localizada y, parcialmente, legitimada por otros (HILBERT, 1984).

Bueno, uno trata de ir al médico para que te dé una solución, pero más o menos todos te dicen lo mismo. Hasta ahora, que por ejemplo, la doctora me dio el tratamiento. (Sonia, 29 años).

\section{Enfrentar los dolores a partir de las señales previas}

Las personas con migraña dijeron reconocer cuándo están por empezar los dolores de cabeza por distintas señales corporales. Algunas de esas señales se repiten en la mayoría de los entrevistados tales como: sensación de abombamiento en la cabeza, náuseas, molestias a la luz, ruido y olores. Otra de las señales (menos frecuente que las anteriores) que adelanta en algunos sujetos la crisis de migraña es el aura. ${ }^{1}$

Lo manejo bien, o sea, aprendí cuando era joven esto que hago ahora, de estar siempre con las cosas encima, porque antes de que me dé el ataque ya me doy cuenta, donde me da la primera señal, ya me enchufo el calmante". (Hilda, 56 años).

Se entiende a los pacientes como sujetos comprometidos y activos en el entendimiento de su padecimiento y en búsqueda de modos de tratamientos que 
le den sentido y encajen con las expectativas culturales y sociales de sus vidas cotidianas (KIRMAYER, 2001). El carácter activo se expresa en estrategias y acciones frente a la cronicidad. Si bien las personas entrevistadas dijeron elegir el sistema biomédico por sobre otras formas de medicina, reconocieron la importancia para ellas de "probar otras cosas por las dudas que alguna haga bien". Así, se ha podido registrar que recurren a yuyos, curanderos, "remedios mágicos", terapia. La mayoría de las personas que recurren a distintas prácticas en búsqueda de alivio del dolor dijeron hacerlo a pesar de saber que "eso a los médicos mucho no les gusta porque no creen”. En los relatos de los sujetos también se encuentran referencias al cansancio que implica convivir con un dolor con el que se debe estar continuamente atento a qué aspecto de la cotidianidad puede ser el desencadenante. Es por eso que el conocimiento de los pacientes respecto de la imposibilidad (al momento) de una cura favorece la discontinuidad en prácticas de cuidado, tratamientos médicos, entre otros.

Tengo una amiga que también tiene dolor de cabeza y estamos siempre con yuyos probando, o sea con el té de tilo, de manzanilla...bueno, que a los médicos mucho no les gusta. No les cae muy bien, pero si buscás en Internet, por ejemplo, algún yuyo que sea para la migraña hay varios. (María, 26 años).

Más allá de las distintas estrategias elegidas, los pacientes relataron que sentían necesidad de "manejar el dolor de la mejor manera posible, aunque los demás no entiendan”.

Trato de no generar acostumbramiento, entonces puedo ir variando...del Flocur... bueno, se me terminó el Flocur, Migral...si no tengo Migral, Cefalex. Fui al psicólogo, hago terapia y me ayuda a descargar un montón de cosas, pero eso no quitó el dolor de cabeza. O sea, la cabeza sigue doliendo igual, pero te ayuda mucho a descargar los nervios, las ansiedades, los traumas infantiles. De hecho también porque la migraña genera cierto trauma. (Paula, 26 años).

Los modelos explicativos de las enfermedades no se presentan de manera coherente sino que dependen, en gran medida, de la interpretación de las personas que están involucradas en su contexto. Se puede decir que en algunas situaciones, las causas objetivas y los diagnósticos formales son menos importantes para las personas que la elaboración de sus propias interpretaciones sobre salud, dolencia, tratamiento, etc. teniendo en cuenta sus experiencias personales y las referencias de su cultura (Caroso et al, 2004). Las personas narran acciones tales como aislarse, recluirse en un cuarto oscuro y silencioso, tomar un medicamento o modificar su 
dosis, cambiar pautas de alimentación, dejar de tomar alcohol, entre otras cosas.

Se trata de respuestas activas frente al padecimiento para intentar modificar, atravesar y convivir con esos dolores. En este sentido, si bien la cotidianidad sufre transformaciones por la dolencia, los dolores de cabeza también son modificados por los sujetos mediante sus dichos y prácticas.

\footnotetext{
Y me conocen la cara, me miran y me dicen: 'Te está doliendo la cabeza'; yo no digo nada a veces y por ahí me miran y se dan cuenta. Pero bueno no es que yo digo: 'Váyanse todos, huyan'. Si me duele, en todo caso me voy yo y me quedo en la habitación, apago las luces y me pongo paños fríos... a veces. (Mirtha, 41 años).

Uno conoce el dolor de cabeza y aprende a manejarlo, digamos. Es un cambio de vida total. Vos los fines de semana en lugar de ir a un shopping, internarte en un lugar cerrado, preferís ir a un lugar abierto. No sé, tratás de tomarte las cosas de otra manera, entonces tratás siempre de priorizarte vos, antes que el otro. (María, 26 años).
}

\section{Espera, paciencia y aguante}

La construcción de la noción de emoción como lo irracional y subjetivo (entre otras características caóticas) juega un papel central en la ideología de las sociedades actuales. Una de las asunciones culturales más importantes es que lo emocional es la antítesis de la razón. La racionalidad se acerca a la inteligencia y la habilidad para resolver problemas. En cambio, las personas tienden a ver lo emocional como lo disruptivo y opuesto al pensamiento. Decir que alguien es emocional es cuestionar la validez de su discurso y, más aún, el sentido de lo que está diciendo (LUTZ, 1986; 1990).

En los relatos sobre los modos en que la migraña interviene en la cotidianidad, existen sensaciones de angustia pero también de acostumbramiento. Los pacientes dijeron aprender a esperar y a tener paciencia. Son formas de convivir y "aguantar" con el padecimiento a largo plazo.

De acuerdo a Scribano, la espera y la paciencia son "mecanismos de soportabilidad social" que refieren a acciones y prácticas hechas cuerpo realizadas (o no) por los sujetos para evitar conflictos sociales (Scribano, 2010). A pesar de las diferencias entre estas investigaciones y los estudios del dolor crónico, estos conceptos permiten examinar ciertas particularidades de la convivencia con el padecimiento de la migraña.

La espera y la paciencia regulan las sensaciones y emociones que atraviesan a los sujetos e indican formas "adecuadas" de comportarse, decir y vivir con 
la dolencia. Se espera que pase el dolor, se tiene paciencia en realizar varias consultas con distintos profesionales, se tiene paciencia en que los tratamientos funcionen y, si no, se transita por distintos consultorios de la misma especialidad en búsqueda de nuevas respuestas.

Recorrí muchísimos neurólogos y la verdad ninguno logró que me calmara mi dolor de cabeza. Siempre tomando las mismas pastillas y nunca un cambio positivo...yo espero que la ciencia siga avanzando y pueda llegar a un buen puerto por el tema de las cefaleas. Cura, por ahora, definitiva creo que no hay... Aparte, el día de mañana me imagino con hijos, y cómo voy a hacer el día que tenga un bebé y llore... y yo voy a estar con dolor de cabeza, vamos a estar los dos llorando (Paula, 26 años).

Las trayectorias de los pacientes con migraña implican, como ellos mismos refieren, un alto grado de "aguante", porque nada garantiza que los tratamientos hagan efecto y que la frecuencia e intensidad de los dolores de cabeza disminuyan. Entre otras cosas, "tener aguante" implica que, a pesar de saber que la migraña no tiene cura y que los tratamientos realizados al momento no tuvieron los efectos esperados, se tiene "paciencia" y se siguen buscando respuestas alternativas. Algunos pacientes entrevistados dijeron consultar a numerosos profesionales por la migraña desde su adolescencia. Incluso conocían los procedimientos y pasos de la consulta. En palabras de uno de ellas: "Creo que en el último año fui a todos los neurólogos para ver qué me hacen, pero cuando les cuento cómo es el dolor y demás, todos me dicen que es migraña" (Paula, 26 años). No obstante, continuaba buscando posibles soluciones porque dijo tener esperanzas de curación en el futuro.

Esperar y tener paciencia son algunas de las formas que tienen los pacientes de "hacer frente" y convivir con la migraña. Para eso, la técnica corporal del aguante no solo modifica los patrones del sentir, sus modos de expresión y la valoración social, sino que produce, reproduce y responde a ciertos mandatos y acusaciones de discursos oficiales y/o dominantes (EPELE, 2010, p. 232).

Las experiencias de salud/dolor son situaciones y acontecimientos de la vida irregulares para los sujetos que no solo afectan su corporalidad más inmediata sino también su vida moral, su auto representación y sus sentimientos (DIAS DUARTE, 2003). Las personas con migraña contaron que uno de los motivos por los cuales tenían que "aguantar y seguir" se relaciona con las características sociales atribuidas a los dolores de cabeza que los vinculan con excusas, exageración y, en algunos casos, mentiras. Es decir, hay un conjunto de discursos 
(biomédicos, sociales, entre otros) que influyen y condicionan a que en algunos padecimientos haya que aguantar y en otros no.

\section{Reflexiones finales}

Al comienzo de este artículo se planteó como interrogante la relevancia que podía tener estudiar algo tan común como los dolores de cabeza. A través de este escrito se intentó mostrar que la migraña es, por lo menos, un dolor complejo, cuyo estudio permite profundizar en las investigaciones sobre dolores crónicos y discutir con alguna de ellas.

En primer lugar, la migraña es un tipo de dolor de cabeza que tensiona las bases tradicionales de la medicina moderna edificada y sostenida por evidencias. ¿Dónde está ubicada la migraña en el cuerpo?, ¿por qué aparece?, ¿por qué algunas personas tienen migraña y otras no?, ¿por qué los tratamientos en general no tienen los resultados esperados? A pesar de los avances dentro de la medicina respecto de la legitimidad de estos dolores de cabeza a través de un diagnóstico y un reconocimiento de la misma como enfermedad, las preguntas realizadas previamente no tienen al momento desde la práctica biomédica respuestas unívocas y certeras.

En segundo lugar, las personas con migraña tienden a ser disruptivas, porque alteran el "orden" de la vida cotidiana caracterizado por la planificación de horarios y tareas. A su vez, no sólo se rompe con lo esperado por avisar siempre a último momento, sino que dadas las significaciones sociales que quienes padecen dicen encontrar en los dolores de cabeza se genera un plus por el cual decir "tengo migraña" no ayuda en la situación. De acuerdo a los relatos de los sujetos, se los tiende a relacionar con "malos hábitos" y "excesos”, como comer de más, dormir de más o de menos, abuso de bebidas alcohólicas, etcétera. Pero, también, se los suele vincular a aquellas personas que son excesivamente responsables. En este último aspecto, el estrés, los nervios y las preocupaciones son elementos que tienden a justificar estos dolores de cabeza.

Un aspecto central que se analizó a través de las nociones de espera y paciencia es que la mayoría de las personas con migraña reconocen que estos dolores se convierten en "algo más con lo que aprenden a convivir", algo que ya está "incorporado". Se trata de un dolor que pasa a formar parte de la biografía personal, porque se tiende a conocer (con asombrosa exactitud) el momento de 
aparición, trayectorias por distintos profesionales y hasta anécdotas detalladas sobre eventos que la aparición del dolor interrumpió. Más aún, seguramente, muchas de las situaciones relatadas no hubieran tenido la relevancia e importancia dada de no ser por encontrarse atravesadas por los dolores de cabeza.

\section{Referencias}

ALABARCES, P.; GARRIGA ZUCAL, J. El "aguante": una identidad corporal y popular. Intersecciones antropología [en línea], n. 9, p. 275-289, 2008.

ALLUÉ, M. La gestión del dolor. In: GRIMBERG, M. (ed.). Experiencias y narrativas de padecimientos cotidianos. Miradas antropológicas sobre la salud, la enfermedad y el dolor crónico. Buenos Aires: Editorial Antropofagia, 2009. p. 167-189.

ALVES, P. A experiência da enfermidade: consideraçōes teóricas. Cad. Saúde Pública. Rio de Janeiro, v. 9, n. 3, p. 263-271, 1995.

ALVES, P.; RABELO, M. Nervios, proyectos e identidades: narrativas de la experiencia. In: GRIMBERG, M. (ed.). Experiencias y narrativas de padecimientos cotidianos: miradas antropológicas sobre la salud, la enfermedad y el dolor crónico. Buenos Aires: Aires: Editorial Antropofagia, 2009.

BERGER, P. LUCKMAN, T. La construcción social de la realidad. Buenos Aires: Amorrortu, 1986.

BUTLER, J. Giving an account of oneself. Diacritics, v. 31, n. 4, p. 22-40, 2001. . Vida precaria. El poder del duelo y la violencia. Buenos Aires: Paidós, 2009.

CASTRO PÉREZ, R. Salud y cotidianidad: un análisis hermenéutico In: GRIMBERG, M. (ed.). Experiencias y narrativas depadecimientos cotidianos: miradas antropológicas sobre la salud, la enfermedad y el dolor crónico. Buenos Aires: Aires: Editorial Antropofagia, 2009. p. 21-52.

CAROSO, C.; RODRIGUES, N.; ALMEIDA-FILHO N. "Nem tudo na vida tem explicação": explorações sobre causas de doença e seus significados. In: LEIBING, A. (Org.). Tecnologias do corpo: uma antropología das medicinas no Brasil. Rio de Janeiro: Nau, 2004. CSORDAS, T. The body as representation and being in the world. In: (Ed.). Embodiment and Experience. The existential ground of culture and self. Cambridge: Cambridge University Press, 1994. p.1-24.

DAS, V. Language and Body: transactions in the construction of pain. In: KLEINMAN, A.;DAS, V. (Eds.). Social Suffering. Berkeley: University of California Press, 2001.

DUARTE, L.F.D. Da vida nervosa nas classes trabalhadoras urbanas. Rio de Janeiro: Jorge Zahar 1986. 
Indivíduo e pessoa na experiência da saúde e da doença. Ciência e Saúde Colectiva.

Rio de Janeiro, v. 8, n. 1, p. 173-183, 2003.

DEL MONACO, R. Dolores de cabeza, síntomas y enfermedad. Un abordaje social sobre la construcción clínica de la migraña. Intersecciones en Antropología, v. 13, p. 501-511, 2012.

DICCIONARIO de la Real Academia Española. Madrid, 201. Disponible en: www.rae.es. Acceso: marzo 7, 2012.

EPELE, M. Sujetar por la herida. Una etnografía sobre drogas, pobreza y salud. Buenos Aires: Paidós, 2010.

FOUCAULT, M. El nacimiento de la clínica. Buenos Aires: Siglo Veintiuno, 2008.

FOUCAULT, M. Historia de la sexualidad. El uso de los placeres. Buenos Aires: Siglo Veintiuno, 2010.

GARRO, L. Chronic illness and the construction of narratives. In: GOOD, B. (Comp.). Pain as Human experience. An anthropological perspective Berkeley: University of California Press, 1994. p. 100-137.

GRIMBERG, M. Narrativas del cuerpo. Experiencia cotidiana y género en personas que viven con VIH. Cuadernos de Antropología Social, Buenos Aires, n. 17, p. 79-99, 2003.

HILBERT, R. The acultural dimension of chronic pain: flawed reality construction and the problem of meaning. Berkeley: University of California Press, 1984.

HELMAN, C. Culture, Health and Illness: An Introduction for Health Professionals. Boston: Wright, 1984.

. Anthropology and clinical practice. Anthropology Today, v. 1, n. 4, p. 7-10, Aug. 1985.

. Dr Frankenstein and the Industrial Body: Reflections on 'Spare Part'. Anthropology Today, v. 4, n. 3, p. 14-16, Jun. 1988.

JACKSON, J. Camp Pain Talking with Chronic Pain Patients. Philadelphia: University of Pennsylvania Press, 2000.

KIRMAYER, L. The body's insistence on meaning: metaphor as presentation and representation in illness experience. Medical Anthropological quarterly New Series, v. 6, n. 4, p. 323-346, 1992.

Cultural variations in the clinical presentation of depression and anxiety: implications for diagnosis and treatment. J Clin Psychiatry, v. 62, supl. 13, p. 22-28, 2001.

. Trauma and disasters in social and cultural context. In: CRAIG, M.; BHUGRA,

D. (Eds). Principles of social psyquiatry. Willey-Blackwell, Oxford, 2010.

KLEINMAN, A. The illness narratives. Suffering, healing anda the human condition. New York: Basic Books, 1988. 

. The Deligitimation and Religitimation of Local Worlds. In: GOOD, B. (Comp.). Pain as Human experience. An anthropological perspective Berkeley: University of California Press, 1994. p. 169-197.

LUTZ, C. Thought, and Estrangement: emotion as a cultural category. Cultural Anthropology, v. 1, n. 3, p. 287-309, Aug. 1986.

. Engendered emotion: gender, power, and the rhetoric of emotional control in

American discourse. In: LUTZ, C; ABU-LUGHOD, L. (Eds.). Language and the politics of emotion. New York: Cambridge University Press, 1990.

MERLEAU PONTY, M. Fenomenología de la percepción. México: Fondo de Cultura Económica, 2003.

OTEGUI PASCUAL, R. El sufrimiento: la forma sociocultural del dolor. In: GRIMBERG, M. (ed.). Experiencias y narrativas de padecimientos cotidianos: miradas antropológicas sobre la salud, la enfermedad y el dolor crónico. Buenos Aires: Aires: Editorial Antropofagia, 2009. p. 147-166.

RABELO, M. C.; ALVES, P. C. Corpo, experiência e cultura. . In: LEIBING, A. (Org.). Tecnologias do corpo: uma antropología das medicinas no Brasil. Rio de Janeiro: Nau, 2004.

RICOEUR, P. Educación y política. Buenos Aires: Educación y Cultura, 1986.

RICOEUR, P. Sí mismo como otro. España: Siglo Veintiuno de España, 1996.

SCARRY, E. The body in pain. New York: Oxford University Press, 1985.

SCRIBANO, A. "Primero hay que saber sufrir...!!! Hacia una sociología de la "espera" como mecanismo de soportabilidad social”. In: SCRIBANO, A.; LISDERO, P. (Eds.). Sensibilidades en juego: miradas múltiples desde los estudios sociales del cuerpo y las emociones. Córdoba: CEA- CONICET E-book, 2010. p. 169-194.

STRAUSS, A. L. et al. Chronic illness and the quality of life. 2a ed. St Louis: Mosby, 1984.

ZAVALA, H.; SARAVIA, B. Los dolores de cabeza. Buenos Aires. Publicación interna del Gabinete de Cefaleas del Hospital Ramos Mejía, 2008.

\section{Nota}

${ }^{1}$ Desde la perspectiva biomédica el aura es una alteración recurrente que se manifiesta por crisis de síntomas neurológicos focales reversibles que usualmente se desarrollan en forma gradual durante 5 a 20 minutos y pueden durar hasta 60 minutos (ZAVALA et al, 2003, p. 72). 
Dor crônica e narrativa: experiências cotidianas e trajetórias de cuidado no sofrimento da enxaqueca

A partir de uma perspectiva sócio-antropológica, o presente trabalho visa contribuir para o conhecimento do sofrimento crônico da enxaqueca. Por esse motivo, foi realizada pesquisa qualitativa, através de entrevistas com médicos neurologistas e pessoas com esta condição em um hospital da Região Metropolitana de Buenos Aires, Argentina. O objetivo principal é explorar, através das narrativas de pessoas que sofrem esta condiçãa, as experiências, práticas cotidianas e trajetórias de cuidado com este tipo de dor de cabeça. Algumas características da enxaqueca, que a diferenciam de outras dores crônicas e influenciam na maneira como os pacientes se relacionam com seu ambiente, são que a enxaqueca não tem condições de verificação factual e que não tem uma etiologia certa nem um tratamento eficaz. A partir disso, os sujeitos dizem que, em alguns casos, o sofrimento é associado à escusas e à falta de legitimidade. Além disso, através das histórias, observa-se que a enxaqueca se torna parte da biografia da pessoa. Finalmente, a maioria dos pacientes diz ter se acostumado a essas dores de cabeça e a ter paciência, algo que influi no relacionamento com si próprio e com os outros.

> Palavras-chave: dor crônica; enxaqueca; experiências corporais; vida cotidiana; trajetórias de cuidado. 
Chronic pain and narrative: everyday experiences and trajectories of care in suffering from migraine

From a socio-anthropological perspective, this paper aims to contribute to the knowledge of suffering chronic migraine. For this reason, qualitative research was conducted through interviews with neurologists and people with this condition in a hospital in the Metropolitan Area of Buenos Aires, Argentina. The main objective is to explore, through the narratives of people suffering this condition, the experiences, daily practices and trajectories of care for this type of headache. Some features of migraine, which differentiate it from other chronic pain and influence the way patients relate to their environment, is that migraine does not allow to check facts and does not have a certain etiology or effective treatment. From this, the subjects say that in some cases, the pain is associated with the excuses and lack of legitimacy. Furthermore, through the stories, it is observed that migraine becomes part of the biography of the person. Finally, most patients said to have become accustomed to these headaches and have patience, something that affects the relationship with themselves and others.

> Key words: chronicle pain; migraine; bodily experiences; daily life; trajectories of care. 